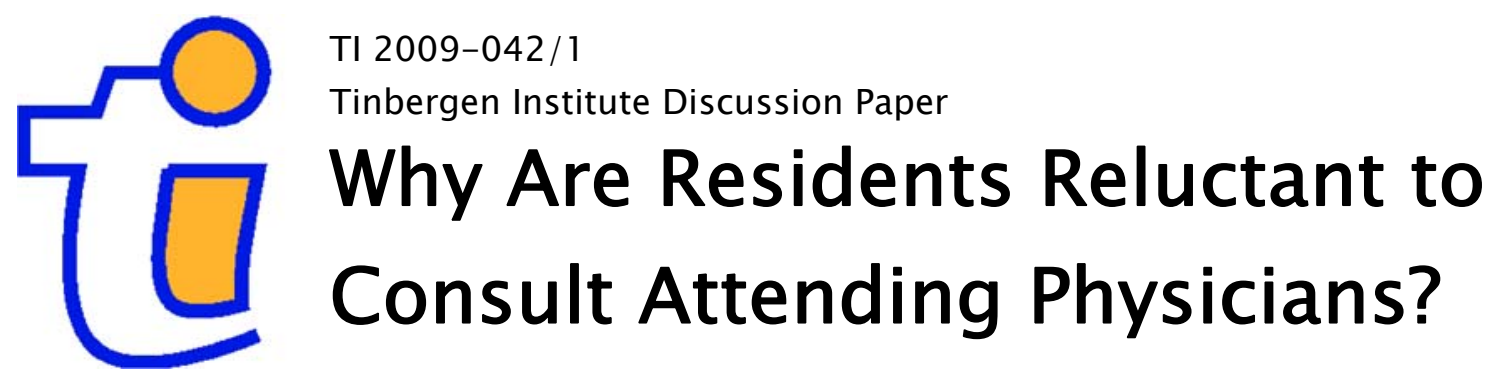

Otto H. Swank

Erasmus School of Economics, Erasmus University Rotterdam, and Tinbergen Institute. 


\section{Tinbergen Institute}

The Tinbergen Institute is the institute for economic research of the Erasmus Universiteit Rotterdam, Universiteit van Amsterdam, and Vrije Universiteit Amsterdam.

Tinbergen Institute Amsterdam

Roetersstraat 31

1018 WB Amsterdam

The Netherlands

Tel.: +31(0)205513500

Fax: $+31(0) 205513555$

Tinbergen Institute Rotterdam

Burg. Oudlaan 50

3062 PA Rotterdam

The Netherlands

Tel.: + $31(0) 104088900$

Fax: $+31(0) 104089031$

Most TI discussion papers can be downloaded at http://www.tinbergen.nl. 


\title{
Why Are Residents Reluctant to Consult Attending Physicians?
}

\author{
Otto H. Swank* \\ Erasmus University Rotterdam \\ and Tinbergen Institute
}

May 13, 2009

\begin{abstract}
A physician performs two tasks: making diagnoses and determining treatments. To reduce medical error, residents are supposed to consult their supervisors when they face uncommon circumstances. However, recent research shows that residents are reluctant to do so. This paper presents a model that explains (i) which residents shy away from consulting; (ii) when residents are reluctant; (iii) the importance of protocols in the medical sector; and (iv) when consulting is a sign of strength or a sign of weakness. Furthermore, I show that encouraging residents to consult by investigating mishaps leads to another distortion: residents will give too much weight to own assessments.
\end{abstract}

Keywords: Medical error, medical decision-making, reputational concerns, consulting.

JEL codes: D21, D82, D83

*Address: Eramus Univeristy Rotterdam, P.O. Box 1738, 3000 DR, Rotterdam, The Netherlands. E-mail: swank@few.eur.nL. I thank Martin B $q$ g, Benoit Crutzen, Josse Delfgaauw and Bauke Visser for helpful comments. 


\section{Introduction}

Suppose you go to a hospital because you have felt ill for a couple of days. A medical resident examines you thoroughly and diagnoses that you suffer from a rare, exotic disease. He proposes a heavy treatment that may lead to problems of infections and depressions. How would you react? As the disease is rare and the treatment is heavy, it is likely that you want the resident to ask a more senior physician to look at you. Before undergoing the heavy treatment, you want the resident's diagnosis to be confirmed.

For many diagnoses it makes sense to ask for a second opinion. The reason is twofold. First, between 44,000 and 90,000 Americans die every year as a result of medical error (Kohn et al., 2000). Diagnosis error is identified as a major source of medical error (Britto and Pamnarayan, 2009). Diagnosis error varies across specializations. Research on second opinion reports percentages of discrepancies between diagnoses in the range of $4 \%$ to $50 \%$ (for an overview see Schiff et al., 2005). Sometimes the second opinion leads to major changes in treatment. For example, for cancer diagnosis in head and neck, $24 \%$ of the changed diagnoses involved a change from a benign to a malignant diagnosis, $15 \%$ involved a change from a malignant to a benign diagnosis, and $61 \%$ involved a change in tumor classification (Westra et al., 2002). Hahm et al. (2001) report similar figures for liver pathology.

The second reason to ask for a second opinion is that physicians themselves are reluctant to consult other physicians in case of uncertainty. This typically applies to residents. Most hospitals have protocols describing the circumstances under which residents should call the attending physician. However, there is strong evidence that residents do not always follow protocols (Sutcliffe et al. 2004). According to Williams et al. (2007) residents' reluctance can be explained by the hierarchical nature of the relationship between the residents and the attending physicians. For the advancement of their study, in particular, and for their career perspectives, in general, residents want to make themselves look good in the eyes of their superiors. Fear for negative feedback discourages residents to consult (Sutcliffe, et al. 2004).

The job of a physician consists of two parts. Making a diagnosis and, on the basis of this diagnosis, determining the treatment. ${ }^{1}$ When a job consists of two

\footnotetext{
${ }^{1}$ Many other jobs also consist of two parts. Consider a book publisher. Part of his job is to
} 
parts, it often raises a matching problem: Doing the job properly requires a good assessment. One way of reducing the probability of incorrect assessments is to allow workers who are uncertain about their assessments to consult more senior workers. Indeed, Garicano (2000) shows that a hierarchy is a natural way to organize the acquisition of knowledge for solving matching problems. The idea is that when an agent faces an uncommon situation he turns to a more senior agent for help or advice. In the case of an hospital, when a resident is uncertain about his diagnosis he should consult an attending physician. Obviously, this way of organizing matching problems requires that residents have proper incentives to consult their superiors. The main objective of the present paper is to investigate those incentives.

Above I have discussed evidence that residents sometimes refrain from consulting in situations where they should do so. It is worth emphasizing that this behaviour is not limited to the medical sector. The business literature on feedback-seeking sheds light on the factors that influence employees' consulting behavior in other working places [for a survey of this literature see Ashford et al. (2003)]. Loosely speaking, in this literature an agent is said to seek feedback when after he has performed (part of) a job, he asks another agent, often a superior, whether or not he is on the right track. ${ }^{2}$ The business literature distinguishes two motives that drive consulting behavior: the instrumental motive and the image-based motive. In this paper we argue that these motives also underlie consulting behavior in the medical sector. The instrumental motive is the point of departure of Section 2. The idea is that a resident seeks feedback to better select the proper treatment for his patient. I show that (i) more uncertainty leads to more consulting; (ii) a resident should call the attending physician if his diagnosis suggests a relatively extreme situation; and (iii) highly able residents seek confirmation from their superiors out of fear of being wrong, whereas less able residents seek feedback out of fear of being right. ${ }^{3}$ Section 2 explains why many hospitals use protocols to guide residents' consulting behavior.

review new manuscripts. On the basis of a review, a decision is made whether or not to publish the book, and how much money to spend on marketing it. The job of a loan officer also consists of two parts. First, he has to determine the creditworthiness of a client and the profitability of an investment plan. After the loan officer has formed a picture of the loan request, he makes a proposal for the type of loan.

${ }^{2}$ We use the words seeking feedback, asking feedback, seeking advice and consulting interchangably.

${ }^{3}$ Ashford and Cummings (1985) and Tuckey et al. (2002) provide evidence supporting these results. The second finding is consistent with Garicano (2000). 
Consulting behavior is also influenced by how residents believe it affects their image; that is, how they are seen by their superiors. ${ }^{4}$ The image motive seems particularly relevant for knowledge-based hierarchies such as exist in hospitals where career concerns are, almost by definition, important. Residents are in training. Attending physicians play an important role in evaluating residents. In Section 3, I present a model in which both the instrumental and the image motive drive consulting behavior. The model explains why protocols that dictate consulting behavior do not always work (Roberts, et al. 2008, and Seilverman et al., 2007). Broadly, the predictions of my model are consistent with the existing evidence on consulting behavior in other workplaces. Ashford and Northcraft (1992) find that fear for negative feedback discourages employees from seeking feedback. On the other flip of the coin, hope for positive feedback is a stimulus for feedback-seeking (Brett et al., 1991). Morrison and Bies (1991) find that the act of seeking feedback and the content of feedback have separate impacts on employees' images. Finally, whether feedback-seeking is a strength or a weakness varies from organization to organization (Ashford et al., 2003).

Section 3 shows that residents may shy away from consulting to conceal their shortcomings from the eyes of others. Abstaining from asking advice increases the likelihood of poor task performance, and may lead to complaints. In Section 4 I examine whether monitoring through investigation of complaints alleviates the distortion in residents' consulting decisions (see, Prendergast, 2003). In the model, monitoring means that the attending observes the resident's diagnosis and how he has acted upon it. I show that monitoring weakens the incentives of less able juniors to distort their consulting decisions. However, monitoring leads to another distortion. It induces residents to give too much weight to their own information. When more able residents should give more weight to their own assessments than less able residents, showing confidence in your own assessment is a sign of strength. Hence, if the senior observes how an employee's action relates to his assessment, reputational concerns lead the employee to attribute too high a weight to his own assessment. The prediction of my model that people tend to overweigh private information is

\footnotetext{
${ }^{4}$ In the economics literature, the image motive is referred to as reputational concerns. Holmström (1999) was one of the first who investigated how reputational concerns can motivate employees.
} 
consistent with findings of social psychologists. Overweighing private information is regarded as one form of overconfidence (see, for example, Healy and Moore, 2007 and Kariv, 2005). In that literature, however, overweighing information is not a choice. People believe that they use information efficiently. Bernardo and Welch (2001) explain in such a context why overconfident individuals survive. They argue that overconfidence is a counterforce to herd behavior. In my model, people are not overconfident in the sense that they truly believe that their information is superior. Rather, residents act as if their information is superior to impress their supervisors.

This paper is closest related to the literature on herding. Scharfstein and Stein (1990) show that in a sequential decision-making process reputational concerns may induce the second player to ignore his private information and to follow the first player. The idea is that signals of able agents are correlated. Agreement therefore signals competence, whereas disagreement signals incompetence (see also Visser and Swank, 2007). In the realm of the herding literature, Ottaviani and Sorensen (2001) addresses the question of the order of speaking. A benefit of letting the more competent agent speak first is that at least the more competent agent's signal is revealed. A benefit of letting the less competent agent to speak first is that that agent has weaker incentives to herd.

My paper deviates from this literature in several respects. Most importantly, the model developed in this paper has interesting predictions in the absence of reputational concerns. It explains why residents should consult their supervisors under uncommon circumstances. In particular the result that an increase in the probability that the resident makes a correct diagnosis may increase the scope of consulting is somewhat surprising. Second, in my model the focus is on the first player, while in the herding literature the focus is usually on the second player. Finally, I do not address the order of play. It is in the nature of the educational process that at a certain time residents are charged with the responsibility to make diagnoses and to determine treatments.

\section{The Instrumental Motive}

\section{Example: dry eye syndrome ${ }^{5}$}

\footnotetext{
${ }^{5}$ See Carrol Patton (2009).
} 
A common eye illness is keraroconjunctivitis, better known as dry eye syndrome. Physicians try to diagnose dry eye in early stages as treatment options rely on how severe the disease is. Depending on the perceived stage of the disease, physicians prescribe specific eye drops. Some drops are prescribed for mild forms of dry eyes, other drops are prescribed for more advanced forms. Occasionally, physicians insert silicane plugs. A physician who has to select a treatment faces the problem that the stage of the disease is hard to diagnose. Tests are often not reliable because eyes can be hypersensitive at different stages: "Symptoms do not always correlate with the stages of disease". As a result, "physicians have to make educated guesses" [Dr. Karpecki, quoted in Patton (2009)].

Our model describes a two-layer hierarchy. On the first layer is a junior physician (one can think of a resident). We refer to this physician as the junior. The junior meets a new patient. The task of the junior is twofold. First, to make a diagnosis, and second, to select a treatment. I model the performance of both tasks as follows. Denote by $x$ the optimal treatment from the patient's perspective. This $x$ is drawn from a distribution function $f(x)$, with $\int_{x} x f(x) d x=\mu$ and $\int_{x}(x-\mu)^{2} f(x) d x=$ $\sigma^{2}$. The patient's preferences are described by

$$
U_{P}=-(e-x)^{2}
$$

where $e$ denotes the treatment selected by the junior. Equation (1) captures that the physician should select the treatment that optimally addresses the patient's problem. The way the junior makes a diagnosis is modelled in a crude way. I assume that the junior receives a signal about $x$. With probability $\pi$ this signal, $s$, is correct, $s=x$. With probability $1-\pi$, the signal is drawn from $f(x)$, but does not contain any information about $x$. The density function $f(x)$ is common knowledge. Before preceeding, let me briefly discuss a well-known illness to which the above way of diagnosing applies.

If the junior does not consult a more senior physician, his treatment decision results from maximizing the expected value of (1), conditional on $s$, with respect to $e$, yielding

$$
e=\pi s+(1-\pi) \mu
$$


Equation (2) shows that the treatment is a weighted average of the junior's signal and the expected value of $x$. As expected, the higher is $\pi$, the higher is the weight the junior attributes to his signal. To reduce notation and without losing any insight into the consulting problem, I assume that $\mu=0$. One can think of $x=0$ as a patient with a common problem (of course in the context of the realm of the disease, say, dry eye syndrome). If $|s|$ is large, I say that the junior's diagnosis suggests an extreme problem. The task requires a treatment that deviates from the standard.

On the second layer is a senior physician. Think of him as the attending physician. By assumption, this senior makes a correct diagnosis. Consulting her, however, entails an opportunity $\operatorname{cost} C$. More specifically, after the junior has received his signal, he can learn the true value of $x$ for sure by consulting the senior at a cost $C{ }^{6}$ The junior's consulting decision $(d \in\{n, y\}$, with $d=n$ denoting "not consulting" and $d=y$ denoting "consulting") is the main focus of the present model. The question I address is for which values of $s$ does the junior consult the senior?

The consulting decision entails a simple cost-benefit analysis. Not consulting yields an expected payoff equal to

$$
\begin{aligned}
U(d=n, s) & =-\pi[\pi s-s]^{2}-(1-\pi) E[\pi s-x]^{2} \\
& =-\pi(1-\pi) s^{2}-(1-\pi) \sigma^{2}
\end{aligned}
$$

where $E$ is the expectation operator. Consulting ensures that the treatment is tailored to the patient's actual problem, $e=x$. The junior consults the senior if the expression in (3) is higher than $-C$ :

$$
S(s)=\pi(1-\pi) s^{2}+(1-\pi) \sigma^{2}-C>0
$$

The function $S(s)$ is a parabola with a minimum at $s=\mu=0$. If $C>(1-\pi) \sigma^{2}$, then the junior does not consult for values of $s$ close to 0 , and consults for values relatively far from 0 . If, by contrast, $C \leq(1-\pi) \sigma^{2}$, then the junior always consults the senior. Proposition 1 summarizes the above discussion.

Proposition 1 Suppose that $C>(1-\pi) \sigma^{2}$ and $0<\pi<1$. The junior does not

\footnotetext{
${ }^{6}$ At the end of this section, I discuss how relaxing the assumption that the senior always makes the correct diagnosis affects the results.
} 
consult $(d=n)$ for values of $s$ relatively close to $\mu$, and consults $(d=y)$ for values of $s$ relatively far from 0 .

Proposition 1 is consistent with the observation discussed in the introduction that consulting takes place when the resident faces an uncommon patient. An implication of Proposition 1 is that for $C>(1-\pi) \sigma^{2}$ two values of $s$ exist, for which a junior is indifferent between consulting and not consulting. Because of our assumption that $\mu=0$, the range of $s$ for which the junior consults can be represented by a single value $\bar{s} \geq 0: S(\bar{s})=S(-\bar{s})=0$, where

$$
\bar{s}=\sqrt{\frac{C-(1-\pi) \sigma^{2}}{\pi(1-\pi)}}
$$

Using (5), one can determine how the junior's inclination to consult depends on the junior's ability of making a correct diagnosis, $\pi$.

Proposition 2 For small values of $\pi$, an increase in $\pi$ shrinks the range of $s$ for which the junior does not consult. For high values of $\pi\left(\pi \geq \frac{1}{2}\right.$ being sufficiently high), an increase in $\pi$ widens the range of $s$ for which the junior does not consult.

To grasp the intuition behind Proposition 2, first suppose that $\pi$ is close to zero, say $\pi=0.1$. Then, the treatment largely depends on $\mu$ and hardly depends on $s$ [see (2)]. If the junior's diagnosis is correct, then the selected treatment deviates much from the patient's problem. So, if $\pi$ is small, the junior runs the danger of making a correct diagnosis. The higher is $\pi$, the larger is this danger. Now suppose that $\pi$ is close to one, say $\pi=0.9$. In such a situation, the selected treatment primarily depends on $s$. For high values of $s$, an uninformative signal would lead to a mismatch between the treatment and the patient's problem. To put it otherwise, the junior runs the danger that he has received a wrong signal. To eliminate this danger, the junior consults the senior for high values of $s$. Clearly, the higher is $\pi$, the lower is the probability of a mismatch.

So far, I have assumed that the junior makes a diagnosis, decides on the treatment and decides on whether or not to consult the senior. Another possibility is that the junior's job description specifies what he should do, and under which circumstances he is regarded to consult the senior. Protocols and job descriptions are important in the medical sector. Consider the following three examples. 
1. $c>(1-\pi) \sigma_{x}^{2}$ and $\pi=0$. In this case, the junior is regarded always to follow the same routine, $e=0$, and never to consult the senior. The task of making a diagnosis does not exist. An example is a nurse who is charged with the task of vaccinating children against measles. In principle, measles vaccine can lead to allergic reactions (for example, children having an allergy to eggs may have an allergic reaction to the MMR vaccination). However, children are rarely subjected to thorough investigation. The cost of an investigation does not outweigh the expected benefits.

2. $c<(1-\pi) \sigma_{x}^{2}$ and $\pi$ being (very) low. In the second case, making a diagnosis and acting upon it are two different jobs. The senior determines what to do, and the junior performs the treatment. In terms of our model, the junior always consults the senior. Division of tasks is very common in Dutch orthodontic centres. Usually, a dentist refers a patient to an orthodontic centre. Next, the orthodontist makes a diagnosis and proposes an orthodontic appliance (often a kind of brace). The brace is finally placed by an assistant. Assistants normally do not have an academic training (low $\pi$ ).

3. $c<(1-\pi) \sigma_{x}^{2}$ and $\pi$ being high. The last example concerns doctors who already had advanced training (higher $\pi$ ). Residents have some freedom to make diagnoses and to make decisions on treatments. However, they are supposed to consult a senior physician under uncommon circumstances and for procedures they cannot perform.

The model presented in this section is the easiest model I could think of to bring home the point that juniors tend to consult seniors under relatively extreme circumstances. Moreover, the model clearly demonstrates two reasons why juniors want to consult. If $\pi$ is small, then a junior consults because he may have received a correct signal. If $\pi$ is high, then a junior runs the danger of having received a wrong signal. An extreme assumption I made is that the senior always make correct diagnoses. Relaxing this assumption does not affect the above results qualitatively either. What matters is that the senior is an additional source of information. For example, I could have assumed that, if consulted, the senior, like the junior, receives a signal that is informative with a certain probability. I would have derived the additional result that the higher is the probability that the senior's signal is correct, the stronger is the junior's incentive to ask feedback. This result is consistent with Fedor et al. (1992), who find that the more credible is the source of information, 
the more likely employees are to seek feedback.

The results presented in the present section are consistent with the literature on second opinion in surgical pathology. Abt et al. (1995) find that in $5.8 \%$ of the cases the second opinion was significantly different from the initial diagnosis. Hahm et al. (2001) find for liver diseases discrepancies in $35 \%$ of the cases. In more than $7 \%$ the change in diagnosis had a significant effect on the chosen treatment. Westra et al. (2002) find major discrepancy rates between initial and second diagnoses of $7 \%$. Many of these changes reflected a change from a benign to a malignant tumor. In their field (beck and head surgery), Westra et al. (2002) recommend a mandatory second opinion for diagnosis leading to demanding treatments. In other words, a physician should consult another physician for diagnoses requiring major treatments.

\section{Reputational Concerns}

As discussed in the introduction, residents have good reasons to make a good impression on their supervisors. In this section, I examine how such reputational concerns affect the resident's decision to consult the attending physician. To this end, I assume that there are two types of juniors, $j \in\{h, l\}$, where $j=h$ denotes that the junior is highly able in making diagnoses, and $j=l$ denotes that the junior is less able in making diagnoses. Similar to the model of the previous section, with probability $\pi_{j}$ the signal a junior of type $j$ receives, $s_{j}$, is correct, $s_{j}=x$. With probability $1-\pi_{j}$, the signal is drawn from $f(x)$, but does not contain any information about $x$. I assume that a highly able junior is more likely to make a correct diagnosis than a less able junior, $\pi_{h}>\pi_{l}$. A junior knows his own ability. The probability that a junior is highly able equals $\rho$. The parameters $\pi_{j}$ and $\rho$ are common knowledge. The junior, whether able or less able, wants to come across as able.

The consulting decision $\left(d_{j}\right)$ contains information about the junior's ability to make correct diagnoses because $d_{j}$ depends on $\pi_{j}$. Throughout this section I assume that $\pi_{l}>\frac{1}{2}$. As shown in the previous section, this assumption implies that less able juniors consult for a wider range of $s$ than more able juniors: $\bar{s}_{h}>\bar{s}_{l}$. Moreover, I assume that in case the junior consults the senior, the latter learns $s$ and how it

relates to $x$. Figure 1 depicts how under these assumptions the junior's consulting 


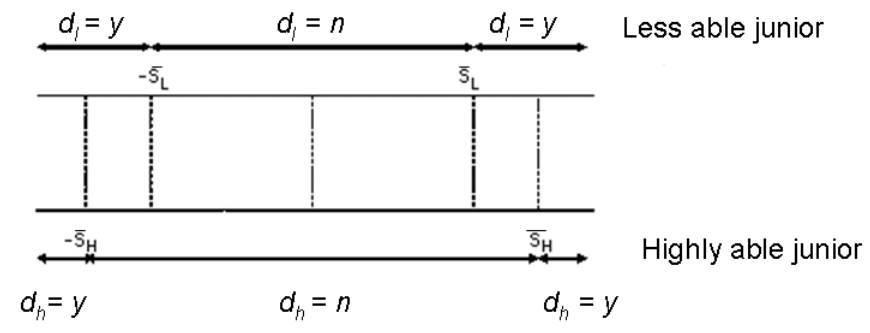

Figure 1: How do the junior's feedback decision depend on his signal?

decision depends on his ability when he is not concerned with his reputation.

Our model is a game of incomplete information. To solve it, I identify perfect Bayesian equilibria, in which the strategies of the two types of juniors are best responses given the posterior probabilities, and the posterior probabilities are updated according to Bayes' rule.

In equilibrium, a junior of type $j$ consults $\left(d_{j}=y\right)$ if [see Equation (4)]

$$
S_{j}\left(s_{j}\right)+\beta[\hat{\rho}(d=y, s, x)-\hat{\rho}(d=n)] \geq 0
$$

where $\hat{\rho}(d=y, s, x)$ denotes the posterior probability that the junior is able, given, $d=y, s$ and $x$; and $\hat{\rho}(d=n)$ is the posterior probability pertaining to the event that the junior does not consult. Let $s_{j}^{*}$ denote the threshold value of $s_{j}$ for which in equilibrium a junior of type $j$ is indifferent between consulting and not consulting [the left-hand side of (6) equals zero].

Lemma 1 In equilibrium, a less able junior consults for a wider range of signals than a highly able junior: $s_{h}^{*}>s_{l}^{*}$.

Proof. The proof is by contradiction. Suppose that $s_{l}^{*} \geq s_{h}^{*}$. An implication is that for $s=\left[s_{h}^{*}, s_{l}^{*}\right), \hat{\rho}(d=y, s, x)=1$. Then, $S_{h}\left(s_{h}^{*}\right)=\beta[\hat{\rho}(d=n)-1]$ and 
$S_{l}\left(s_{h}^{*}\right) \leq \beta[\hat{\rho}(d=n)-1]$, so that $S_{l}\left(s_{h}^{*}\right) \leq S_{h}\left(s_{h}^{*}\right)$. However, the assumption that $\pi_{l}>\frac{1}{2}$ ensures that $S_{l}\left(s_{h}^{*}\right)>S_{h}\left(s_{h}^{*}\right)$. To see this, differentiate (5) with respect to $\pi$.

Lemma 1 has a couple of implications for how consulting affects the senior's perception of the junior's ability. First, not consulting improves a junior's reputation:

$$
\hat{\rho}(d=n)=\frac{\rho s_{h}^{*}}{\rho s_{h}^{*}+(1-\rho) s_{l}^{*}}>\rho
$$

Equation (7) directly follows from Lemma 1. As in equilibrium, highly able juniors consult less frequently than less able juniors, not consulting increases the posterior probability that the junior is highly able (relative to the prior). Second, when $s$ lies in either of two intervals, a less able junior reveals his type by consulting:

$$
\hat{\rho}\left(d=y, s_{l}^{*}<s<s_{h}^{*}, x\right)=\hat{\rho}\left(d=y,-s_{h}^{*}<s<-s_{l}^{*}, x\right)=0
$$

The reason for this result is that in the specified intervals a highly able junior does not consult, whereas a less able junior does. Consulting is therefore a clear sign of being less able. Finally, if $s>s_{h}^{*}$ (or $s<s_{h}^{*}$ ), then the act of consulting does not contain information about the junior's type. As $\pi_{h}>\pi_{l}$, whether or not the diagnosis is correct does contain information about the junior's ability:

$$
\begin{aligned}
& \hat{\rho}\left(d=y, s<-s_{h}^{*} \vee s>s_{h}^{*}, s=x\right)=\frac{\rho \pi_{h}}{\rho \pi_{h}+(1-\rho) \pi_{l}} \\
& \hat{\rho}\left(d=y, s<-s_{h}^{*} \vee s>s_{h}^{*}, s \neq x\right)=\frac{\rho\left(1-\pi_{h}\right)}{\rho\left(1-\pi_{h}\right)+(1-\rho)\left(1-\pi_{l}\right)}
\end{aligned}
$$

The expected reputation of a junior of type $j$ when consulting in these cases equals

$$
\begin{aligned}
& \hat{\rho}_{j}^{e}\left(d=y, s<-s_{h}^{*} \vee s>s_{h}^{*}\right) \\
= & \pi_{j} \frac{\rho \pi_{h}}{\rho \pi_{h}+(1-\rho) \pi_{l}}+\left(1-\pi_{j}\right) \frac{\rho\left(1-\pi_{h}\right)}{\rho\left(1-\pi_{h}\right)+(1-\rho)\left(1-\pi_{l}\right)}
\end{aligned}
$$

Notice that the expected reputation of a highly able junior is better than that of a less able one, as $\pi_{h}>\pi_{l}$.

With the help of (7-11), I can identify two equilibria of the consulting game. ${ }^{7}$

\footnotetext{
${ }^{7}$ For sufficient high values of $\beta$, an equilibrium exists in which both types of juniors always
} 
Proposition 3 describes them.

Proposition 3 (A) Suppose that $\hat{\rho}_{h}^{e}\left(d=y, s<-s_{h}^{*} \vee s>s_{h}^{*}\right) \leq \hat{\rho}(d=n)$. Then, an equilibrium exists in which

(i) a junior of type $l$ consults if $s_{l} \geq s_{l}^{*}>\bar{s}_{l}$, with $s_{l}^{*}$ solving

$$
S_{l}\left(s_{l}^{*}\right)=\beta \hat{\rho}(d=n),
$$

(ii) a junior of type $h$ consults if $s_{h} \geq s_{h}^{*}>\bar{s}_{h}$, with $s_{h}^{*}$ solving

$$
S_{h}\left(s_{h}^{*}\right)=\beta\left[\hat{\rho}(d=n)-\hat{\rho}_{h}^{e}\left(d=y, s<-s_{h}^{*} \vee s>s_{h}^{*}\right)\right]
$$

(iii) a junior of type $j$ chooses effort $e_{j}=\pi_{j} s_{j}$ and

(iv) the posterior probabilities are given by (7-11).

(B) Now suppose that $\hat{\rho}_{h}^{e}\left(s<-s_{h}^{*} \vee s>s_{h}^{*}\right)>\hat{\rho}(d=n)$. Then, an equilibrium exists in which (i), (iii) and (iv) hold. Moreover, a junior of type $h$ consults if $s_{h} \geq s_{h}^{*}=\bar{s}_{h}$.

The first part of Proposition 3 describes an equilibrium in which both types of juniors distort the consulting decision. Reputational concerns induce both types to abstain from consulting in situations where consulting is desirable. Notice that the right-hand side of (12) is higher than the right-hand side of (13). This means that at the margin the drop in reputation resulting from consulting is larger for the less able junior than for the more able one.

The second part of Proposition 3 describes an equilibrium in which only less able juniors distort the consulting decision. To understand why a highly able junior does not necessarily have an incentive to distort, first notice that

$$
\hat{\rho}_{h}^{e}\left(d=y, s<-s_{h}^{*} \vee s>s_{h}^{*}\right)>\hat{\rho}(d=n)
$$

implies that a distortion would go into the direction of more consulting rather than less consulting. However, for $s_{h}$ just smaller than $s_{h}^{*}$, consulting would make the

consult $\left(s_{l}^{*}=s_{h}^{*}=0\right)$ with posteriors probabilities given by (9) and (10), and an out of equilibrium belief $\hat{\rho}\left(d_{j}=n\right)=0$. 
senior believe that the junior is less able. ${ }^{8}$

Identifying the conditions under which one of the two equilibria described in Proposition 3 occurs amounts to determining for which parameters consulting yields a better reputation for the senior than not consulting. One important determinant is how much $\pi_{h}$ deviates from $\pi_{l}$. If a highly able junior makes a correct diagnosis with a much higher probability than a less able junior, then making a correct diagnosis is a strong signal of being highly able. As a result the inequality $\hat{\rho}_{h}^{e}\left(d=y, s<-s_{h}^{*} \vee s>s_{h}^{*}\right)>\hat{\rho}(d=n)$ is likely to hold. The same is true for large values of $\beta$. I have shown that especially less able juniors have strong incentives to abstain from consulting. However, the more a less able junior distorts the consulting decision, the less consulting signals ability. After a certain point, highly able juniors boost their reputations by consulting instead of abstaining from consulting.

As discussed in the introduction, in the medical sector residents have often been found reluctant to consult their supervisors out of fear of coming across as ignorant. More general, several studies report that fear for negative feedback discourages consulting [see the references in the survey by Ashford et al. (2003)]. In my model, less able juniors expect to damage their images by consulting. Highly able juniors, by contrast, may enhance their images by consulting. Therefore, reputational concerns especially make less able juniors more reluctant to consult. However, in spite of this result, less able juniors keep consulting more frequently than highly able juniors (see Lemma 1). Morrison and Bies (1991) find that consulting affects individuals in two different ways: by the act of consulting as well as by its content. Basically, (7-11) tell the same story. Equations (7) and (8) refer to the effect of the act of consulting on a junior's image, while (9-11) refer to its content.

My model sheds light on the question to what extent consulting is considered as a sign of strength. I find that in expectations not consulting improves a junior's reputation [see (7)]. However, the more important are reputational concerns, the less abstaining from consulting enhances a junior's reputation (cf. Ashford and

\footnotetext{
${ }^{8}$ The equilibrium described by the second part of Proposition 3 is not unique. To see this, suppose that $s_{h}^{*}$ is just below $\bar{s}_{h}$ and that $s_{l}^{*}$ solves (12). The posterior probabilities corresponding to these strategies are such that a smart agent does not want to deviate [provided $\left.\hat{\rho}_{h}^{e}\left(s<-s_{h}^{*} \vee s>s_{h}^{*}\right)>\hat{\rho}(d=n)\right]$. In fact, any pair of strategies, $s_{l}^{*}$ solving (12) and $s_{h}^{*}>s_{h}^{\prime}$ where $s_{h}^{\prime}$ solves $\hat{\rho}_{h}^{e}\left(s<-s_{h}^{\prime} \vee s>s_{h}^{\prime}\right)=\hat{\rho}(d=n)$ ], can be part of an equilibrium. The equilibrium described in Proposition 3 is the only one in which a smart agent does not distort the feedback decision.
} 
Northcraft, 1992). Moreover, the question whether consulting is a weakness or a strength cannot be isolated from the question of the expected content of consulting. For a less able junior, not consulting is a strength. It enhances his image. For a highly able junior, consulting may improve his image (in expected terms).

\section{Monitoring by investigation}

In the model of Section 3 patients are sometimes treated poorly. In practice, poor task treatment may lead to complaints. Complaints, in turn, may induce supervisors to investigate cases in more detail. Indeed, investigation of complaints is a wellknown way of monitoring agents. In the present section, I examine how investigation of complaints affects consulting behaviour.

More specifically, I extend the model of the previous section in the following way. I assume that if a mismatch between the treatment and the disease occurs and it exceeds a certain value, $\left|e_{j}-x\right|>v$, the senior receives a complaint and investigates the case. ${ }^{9}$ By investigating the senior learns the junior's diagnosis, the treatment, and the disease. In the model of the previous sections, a mismatch between the treatment and the disease could occur for two reasons. The first reason is that the agent makes a wrong diagnosis and did not consult the senior. The second reason is that the junior made the correct diagnosis, but put too little weight to it: $\left|\pi_{j} s_{j}-s_{j}\right|>v$. By sometimes investigating cases, the senior does not want the junior to put more weight on his signal (although below I will argue that the junior does). Rather, she wants the junior consult more often. To focus on the question how monitoring affects the junior's consulting decision I assume that the highest (lowest) value $x$ can take equals $h(-h)$, such that $\left|\pi_{l} h-h\right|<v$. This restriction ensures that if a junior receives a correct signal and acts upon it in line with (2), it never leads to a complaint. Furthermore, to keep things simple I assume that $f(x)$ is a uniform distribution function with range $2 h$.

Before presenting the equilibrium of the present model, it is fruitful to discuss why the equilibria presented in Proposition 3 do not survive when the senior some-

\footnotetext{
${ }^{9}$ In many settings, it is more plausible to assume that the senior may receive a complaint only if too little effort has been put in the task. Allowing for this asymmetry does not affect the results qualitatively.
} 
times monitors. The reason is that introducing monitoring by investigation into the model turns the model into a signalling game. To see this, suppose that a less able junior receives a positive signal $s_{l}$ that is close to zero. Then, in the equilibria of Proposition 3 this junior does not consult the senior, and exerts effort $e_{l}=\pi_{l} s_{l}$. With some positive probability the senior investigates the case and learns the relationship between $e_{l}$ and $s_{l}$. From this relationship she infers that the junior is of the less able type. By contrast, if the senior were to learn that $e_{h}=\pi_{h} s_{h}$, she would infer that the agent is highly able. The implication is that a less able junior has an incentive to mimic a highly able junior. He can do so by attributing a high weight to his signal $\left(e_{l}=\pi_{h} s_{l}\right)$. The other flip of the coin is that a highly able junior wants to distinguish himself from a less able junior. As a result, the highly able junior also has an incentive to attribute too high a weight to his signal $\left(e_{h}>\pi_{h} s_{h}\right)$.

To present an equilibrium of this game we need some additional notation. I denote by $m=y$ the event that the senior monitors and by $m=n$ the event that the senior does not monitor. Moreover, I denote by $P_{l}$ the probability that the senior monitors, $\operatorname{Pr}(m=y \mid j)=P_{j}$. Finally, I assume that $e_{j} \leq s_{j}$. The implication of this assumption is that the junior cannot attribute a weight higher than one to his signal. In this section I limit attention to the equilibrium in which both types of juniors tend to abstain from consulting. As in the model of the previous section, a second equilibrium exists in which only the less able junior distorts the consulting decision (see part B in Proposition 3).

Proposition 4 An equilibrium of the consulting game with monitoring exists in which:

(i) a junior of type $l$ seeks feedback if $s_{l} \geq s_{l}^{*}>\bar{s}_{l}$, where $s_{l}^{*}$ solves

$$
S_{l}\left(s_{l}^{*}\right)=\beta\left[1-P_{l}\right] \hat{\rho}(d=n, m=n),
$$

and $P_{l}=\left(1-\pi_{l}\right) \frac{1}{2 h}(2 h-2 v)$.

(ii) a junior of type $h$ consults if $s_{h} \geq s_{h}^{*}>\bar{s}_{h}$, where $s_{h}^{*}$ solves

$$
S_{h}\left(s_{h}^{*}\right)=\beta\left[\left(1-P_{h}\right) P \hat{\rho}(d=n, m=n)+P_{h}-\hat{\rho}_{h}^{e}\left(s<-s_{h}^{*} \vee s>s_{h}^{*}\right)\right],
$$

and $P_{h}=\left(1-\pi_{h}\right) \frac{1}{2 h}(2 h-2 v)$. 
(iii) a junior of type $l$ chooses the treatment $e_{l}^{*}=s_{l}$ if $s_{l} \leq \tilde{s}_{l}=\frac{\sqrt{\beta P_{l} \rho}}{1-\pi_{l}}$, and chooses $e_{l}^{*}=\pi_{l} s_{l}$ otherwise; $\tilde{s}_{l}<s_{l}^{*}$.

(iv) a junior of type $h$ chooses the treatment $e_{h}^{*}=s_{h}$ if $s_{h} \leq \frac{\sqrt{\beta P_{l} \rho}}{1-\pi_{l}}$, chooses $e_{h}^{*}=$ $\max \left\{\pi_{h} s_{h}, e_{h}^{m}\right\}$ where $e_{h}^{m}$ solves

$$
\pi_{l}^{2} s_{l}^{2}=-e_{h}^{2}+2 \pi_{h} e_{h} s_{l}+\left(1-\pi_{l}\right) \beta P \rho
$$

if $\frac{\sqrt{\beta P_{l} \rho}}{1-\pi_{l}}<s_{h}<s_{l}^{*}$, and chooses $e_{h}^{*}=\pi_{h} s_{h}$ otherwise.

(v) Posteriors: $\hat{\rho}\left(d=n, m=y, s \leq \frac{\sqrt{\beta P_{l} \rho}}{\left(1-\pi_{l}\right)}\right)=\rho$

$\hat{\rho}\left(d=n, m=y, e=\pi_{l} s_{l}\right)=0$

$\hat{\rho}\left(d=n, m=y, e=\max \left\{e_{m}, \pi_{h} s_{h}\right\}, s>\frac{\sqrt{\beta P_{l} \rho}}{\left(1-\pi_{l}\right)}\right)=1$

$\hat{\rho}(d=n, m=n)=\frac{\rho s_{h}^{*}}{\rho s_{h}^{*}+(1-\rho) s_{l}^{*}}$

$\hat{\rho}\left(s_{l}^{*}<s<s_{h}^{*}\right)=0$.

The posteriors $\hat{\rho}\left(s<-s_{h}^{*} \vee s>s_{h}^{*}, s=x\right)$ and $\hat{\rho}\left(s<-s_{h}^{*} \vee s>s_{h}^{*}, s \neq x\right)$ are given by (9) and (10), respectively.

\section{Proof. See Appendix}

Proposition 4 shows that monitoring has two main effects. First, monitoring leads to a distortion of the effort decision. The reason for this effect is that without monitoring the junior's effort is a weighted average of his signal and the expected difficulty of the task with a highly able junior attributing a higher weight to his signal than a less able junior. Monitoring provides an incentive to a junior to pretend to be highly able by choosing an effort level that depends to a large extent on his signal. For low values of $s_{j}$, the cost of attributing a high weight to one's signal is small. It does not have a substantial effect on the junior's effort level. As a result, for small values of $s_{j}\left(s_{j} \leq \tilde{s}_{l}\right)$, both less able and highly able juniors base their effort decision solely on their signals $\left(e_{j}=s_{j}\right)$. For larger values of $s_{j}$, completely ignoring the possibility that you are wrong is too costly. In expectations, it leads to a too large mismatch between the effort level and the difficulty of the task. Attributing too high a weight to one's signal is more expensive for a less able junior than for a highly able junior. The implication is, as in many other signaling games, that when $s_{h}>\tilde{s}_{l}$ the more able junior chooses an effort level that just discourages a less able junior from mimicking. The above behavior has obvious implications for a junior's reputation 
when the senior monitors. For small values of $s_{j}$, the junior's effort level and signal do not provide information on the junior's type. The posterior probability that the junior is highly able remains $\rho$. For higher values of $s_{j}$, the senior learns the junior's type by monitoring. As discussed in the introduction, the idea that agents too much emphasize private information is not novel in social sciences. In the psychological literature, this is one form of overconfidence. However, in our model juniors are not overconfident in the sense that they really believe that they possess superior information. Juniors pretend to have superior information to signal their ability. ${ }^{10}$

Second, monitoring directly and indirectly affects the consulting decision. The direct effect is simple. The incentive for a less able junior not to consult stems from mimicking a highly able junior. By monitoring the senior may learn the junior's type. As a result, monitoring weakens a less able junior's incentive not to consult. The opposite is true for a highly able junior, as such a junior wants the senior to learn his type. The indirect effects of monitoring on the consulting decision run through the posterior probabilities. These effects are more complicated. On the one hand, the direct effect of the possibility of monitoring induces less able juniors to consult more and more able juniors to consult less. The posterior corresponding to the event that the senior does not monitor therefore rises. However, if the senior monitors, he either learns nothing about the junior's reputation or learns his type. In general, this is good for the reputation of a highly able junior, but bad for a less able one. Overall, the indirect effects may alleviate or aggravate the distortion in the consulting decision.

So far in this section I have assumed that $f(x)=\frac{1}{2 h}$. As discussed before, the implication of this assumption is that the consulting decision does not affect the probability of a complaint. In many situations, a more plausible assumption is that the consulting decision does affect the probability of a complaint. For example, suppose that $f(x)$ is a symmetric function with a maximum at $x=0$. Then, if $s_{j}$ is incorrect and the junior does not consult, a higher value of $s_{j}$ increases the probability that $\left|e_{j}-x\right|>v$. Consequently, at the margin not consulting increases the probability of a complaint. Clearly, in our model less able juniors fear complaints

\footnotetext{
${ }^{10}$ Recall that just above Proposition 4, I have assumed that $e_{j} \leq s_{j}$. Dropping this assumption affects equilibrium behaviour for small values of $s_{j}$. Less able junior will choose $e_{l}=\pi_{l} s_{l}$, and highly able juniors choose effort in accordance with (16).
} 
more than highly able juniors. The reason is that for $s_{j}>\tilde{s}_{l}$, investigation of a complaint reveals the junior's type. From this perspective, a less able junior suffers from a complaint, but a highly able junior benefits. The assumption that $f(x)$ is uniformly distributed is therefore not innocuous. Relaxing it affects a junior's consulting decision.

The upshot of this section is that monitoring juniors by investigating complaints as a means of guiding juniors' consulting decision has an important drawback. It leads to another distortion. The intuition is clear. Without monitoring, the consulting decision was the only opportunity for a junior to affect his reputation. In Section 3 , this led juniors to distort the consulting decision. The problem with monitoring is that it enables juniors to boost their reputation through the effort decision. In our model, without monitoring juniors do not distort the effort decision. Private information is optimally utilized. Monitoring gives incentives to give too much weight to private information.

\section{Conclusion}

A physician's job consists of two parts. First, he must make a diagnosis, and second, he must determine the treatment. In the present paper, I have modeled both tasks as a matching problem: the treatment should be tailored to the diagnosis. In the model an incorrect diagnosis usually leads to a mismatch between the treatment and the disease. I have examined the incentives of residents to consult their supervisors. I have shown that residents should consult when they assess that the disease is uncommon. The reason for this finding varies from environment to environment. In case it is likely that a diagnosis is correct, a resident should consult because his diagnosis might be incorrect. This would lead to a big mismatch between the treatment and the disease. By contrast, when the probability of a correct diagnosis is low, a resident should consult for an uncommon task because he might be correct.

Furthermore, I have shown that reputational concerns may lead to a distortion in the consulting decision. As highly able residents tend to consult less frequently than less able residents, consulting damages reputations. Less able residents distort the consulting decision to a larger extent than highly able residents. Finally, I have shown that monitoring through investigating complaints about poor treatments has 
an important drawback. It gives incentives to residents to attribute too much weight on private information.

\section{Appendix}

Proof of Proposition 4. The proof consists of four steps. First, I focus on the effort decision of a less able junior. Second, I discuss the consulting decision of the less able junior, and show that $s_{l}^{*}>\tilde{s}_{l}$. Next, I determine the effort a highly able junior puts in the task. Finally, I return to the consulting decision of a highly able junior. The posterior probabilities directly result from the strategies.

\subsection{Determination of $e_{l}$}

I have to show that if a junior of type $l$ does not consult and $s_{l} \leq \tilde{s}=\frac{\beta P_{l} \rho}{\left(1-\pi_{l}\right)}$, then $e_{l}=s_{l}$. The benefit of choosing $e_{l}=s_{l}$ rather than choosing $e_{l}=\pi_{l} s_{l}$ is that monitoring does not lead to a drop in the junior's reputation. The cost is a distorted effort decision. At $\tilde{s}=\frac{\beta P_{l} \rho}{\left(1-\pi_{l}\right)}$, the benefit is equal to the cost: $e_{l}=\pi_{l} s_{l}$ yields a payoff:

$$
-\pi_{l}\left(1-\pi_{l}\right) s_{l}^{2}-\left(1-\pi_{l}\right) \sigma^{2}+\left[\pi_{l}+\left(1-\pi_{l}\right)\left(1-P_{l}\right)\right] \hat{p}(d=n, m=n)
$$

and $e_{l}=s_{l}$ yields:

$$
-\left(1-\pi_{l}\right) E\left(s_{l}-x\right)^{2}+\left[\pi_{l}+\left(1-\pi_{l}\right)\left(1-P_{l}\right)\right] \hat{p}(d=n, m=n)+\left(1-\pi_{l}\right) \beta P_{l} \rho
$$

Equating both expressions and solving for $s_{l}$ yields $s_{l}=\tilde{s}_{l}=\sqrt{\frac{\beta P_{l} \rho}{1-\pi_{l}}}$. As the cost of $e_{l}=s_{l}$ increases in $s_{l}$ while the reputational benefits are independent of $s_{l}$, the junior chooses $e_{l}=s_{l}$ if $s_{l} \leq \tilde{s}_{l}$.

\subsection{The consulting decision of a junior of type $l$, and $\tilde{s}_{l}<s_{l}^{*}$}

It is easy to see that given the posterior probabilities Lemma 1 also holds for the consulting game with monitoring. The implication is that when $s_{j}=s_{l}^{*}$, consulting damages a junior's reputation in expected terms. Specifically, a junior of type $l$ 
consults if $s_{l}>s_{l}^{*}$ where $s_{l}^{*}$ solves

$$
S_{l}\left(s_{l}^{*}\right)=\beta\left[1-P_{l}\right] \hat{\rho}(d=n, m=n)
$$

To prove that $\tilde{s}_{l}<s_{l}^{*}$, we must show that $S_{l}\left(\tilde{s}_{l}\right)-\beta\left[1-P_{l}\right] \hat{\rho}(d=n, m=n)<0$ or

$$
\begin{aligned}
& \pi_{l}\left(1-\pi_{j}\right) \tilde{s}_{l}^{2}+\left(1-\pi_{l}\right) \sigma^{2}-C-\beta\left[1-P_{l}\right] \hat{\rho}(d=n, m=n) \\
= & \pi_{l} \beta P_{l} \rho+\left(1-\pi_{l}\right) \sigma^{2}-C-\beta\left[1-P_{l}\right] \hat{\rho}(d=n, m=n)<0
\end{aligned}
$$

(where I have used that $\left.\tilde{s}_{l}^{2}=\frac{\beta P_{l} \rho}{1-\pi_{l}}\right)$. The expression $\left(1-\pi_{j}\right) \sigma^{2}-C$ is by assumption smaller than zero. Moreover, as $\hat{\rho}(d=n, m=n)>\rho$, it suffices to show that $\pi_{l} P_{l}-\left(1-P_{l}\right)<0 . \quad P_{l}=\left(1-\pi_{l}\right) \frac{1}{2 h}(2 h-2 v)$ implying $\pi_{l} P_{l}-\left(1-P_{l}\right)=$ $\left(1-\pi_{l}^{2}\right) \frac{1}{2 h}(2 h-2 v)-1<0$, which is always true.

\subsection{Determinaton of $e_{h}$}

If $s_{l} \leq \tilde{s}_{l}$, a junior of type $h$ cannot distinguish himself from a low ability type. A plausible out of equilibrium belief is: $\hat{\rho}\left(s_{j} \leq \tilde{s}_{l}, e_{j}<\pi_{j} s_{j}\right)=0$. Then, $e_{h}=s_{h}$.

Now suppose $s_{l}>\tilde{s}_{l}$. Then a junior of type $h$ can distinguish himself by choosing an effort level a junior of type $l$ does not want to mimic. Choosing $e_{l}=\pi_{l} s_{l}$ yields a payoff to a junior of type $l$ equal to

$$
-\pi_{l}\left(1-\pi_{l}\right) s_{l}^{2}-\left(1-\pi_{l}\right) \sigma^{2}+\left[\pi_{l}+\left(1-\pi_{l}\right)\left(1-P_{l}\right)\right] \hat{p}(d=n, m=n)
$$

Choosing $e_{l}=e_{h}$ would yield

$$
\begin{aligned}
& -\pi\left(e_{h}-s_{l}\right)^{2}-(1-\pi) E\left(e_{h}-x\right)^{2}+\left[\pi_{l}+\left(1-\pi_{l}\right)\left(1-P_{l}\right)\right] \hat{p}(d=n, m=n) \\
& +\left(1-\pi_{l}\right) \beta P_{l} \rho
\end{aligned}
$$

Equating the above equation yields

$$
\pi_{l}^{2} s_{l}^{2}=-e_{h}^{2}+2 \pi_{h} e_{h} s_{l}+\left(1-\pi_{l}\right) \beta P \rho
$$

By choosing the effort level that solves this equation $\left(e_{h}^{m}\right)$, a junior of type $h$ distinguish himself from a junior of type $l$. Of course, if $\pi_{h} s_{h}>e_{h}^{m}$, the junior of type $h$ 
chooses $e_{h}=\pi_{h} s_{h}$. Hence, for $s_{h}>\tilde{s}_{l}, e_{j}=\max \left\{e_{m}, \pi_{h} s_{h}\right\}$.

\subsection{The consulting decision of a junior of type $h$}

Equating the payoff to the senior when consulting and not consulting yields:

$$
S_{h}\left(s_{h}^{*}\right)=\beta\left[\left(1-P_{h}\right) P \hat{\rho}(d=n, m=n)+P_{h}-\hat{\rho}_{h}^{e}\left(s<-s_{h}^{*} \vee s>s_{h}^{*}\right)\right]
$$

as in Proposition 4.

\section{References}

Abt, A.B., L.G. Abt and G.J. Olt, 1995, The Effect of Interinstitution Anatomic Pathology Consultation on Patien Care, Archives of Pathology and Laboratory Medicine, 119, 514-517,.

Ashford, S. J., R. Blatt and D. Vande Walle, 2003, Reflections on the Looking Glass: A Review of Research on Feedback-Seeking Behavior in Organizations, Journal of Management, 29, 773-799.

Ashford, S., and G.B. Northcraft, 1992, Conveying More (or Less) than We Realize: The Role of Impression Management in Feedback Seeking, Organizational Behavior and Human Decision Processes, 53, 310-334.

Bernardo A.E. and I. Welch, 2001, On the Evolution of Overconfidence and Entrepeneurs, Journal of Economics and Management Strategy, 20, 301-330.

Brett, J.M., D.C. Feldman and L.R. Weingart, 1990, Feedback-seeking Behavior of New Hires and Job Changers, Journal of Management, 16, 737-749.

Britto, J. and P. Ramnarayan, 2006, ISABEL Interfaced with EMR - Impact on diagnosis error, patient, safety and quality of care, The Informatics Review, 9, 13.

Fedor, D.B., R.B. Rensvold, S.M. Adams, 1992, An Investigation of Factors Expected to Affect Feedback Seeking: A Longitudinal Field Study, Personnel Psychology, 45, 779-805.

Garicano, L., 2000, Hierarchies and the Organization of Knowledge in Production, Journal of Political Economy, 108, 874-904. 
Hahm, G.K., T.H. Niemann, J.G. Lucas, and W.L. Frankel, 2001, The Value of Second Opinion in Gastrointestinal and Liver Pathology, Archives of Pathology and Laboratory Medicine, 125, 736-739.

Healy, P.J., and D.A. Moore, 2007, Bayesian Overconfidence, mimeo.

Holmström, B., 1999, Managerial Incentive Problems: A Dynamic Perspective, Review of Economic Studies, 66, 169-182.

Kariv, S., 2005, Overconfidence and Informational Cascades, UC Berkeley, mimeo. Kohn, L.T., J.M. Corrrigan, and M.S. Donaldson, 2000, To Err is human: Building a Safer Health system. Committee on Quality of Health Care in America, Institute of Medicine. Washington D.C: National Academy Press.

Morrison, E.W., and R.J. Bies, 1991, Impression Management in the Feedbackseeking Process: A Literature Review and Research Agenda, The Academy of Management Review, 16, 522-541.

Ottaviani, M and P. Sorensen, 2001, Information Aggregation in Debate: Who Should Speak First?, Journal of Public Economics, 81, 393-421.

Patton, C., 2009, Dry Eye Treatment Should Be Tailored to Patient, Modern Medicine, 15 February.

Prendergast, C., 2003, The Limits of Bureaucratic Efficiency, Journal of Political Economy, 111, 929-958.

Roberts, M., G. Tabas, F. Rubin, S. Bowser and G. Arnold, 2008, Communication Between Attendings and Residents-Differences in Expectations, Journal of General Internal Medicine, 12 Issue Supplement 1, Pages 97 - 97.

Scharfstein, D.S., and J.C. Stein, 1990, Herd Behavior and Investment, American Economic Review, 80, 465-479.

Schiff, G.D., Kim S., Abrams R., et al., 2005, Diagnosing diagnosis errors: Lessons from a multi-institutional collaborative project. Advances in patient safety: From research to implementation. Vol. 2, Concepts and methodology. AHRQ Pub. 050021-2. Rockville, MD: Agency for Healthcare Research and Quality.

Silverman, A.T., M.A. Goldfarb, and T. Baker, 2009, When Should a Surgical Resident Call an Attending Surgeon?, Journal of Surgical Education, 65, 206-212. 
Sutcliffe, K.M., Lewton, E, Rosenthal, M.M, 2004, Communication Failures: An Insidious Contributor to Medical Mishaps, Academic Medicine, 79, 186-194.

Tuckey, M., N. Brewer and P. Williamson, 2002, The Influence of Motives and Goal Orientation on Feedback Seeking, Journal of Occupational and Organizational Psychology, 75, 195-216.

Visser, B. and O.H. Swank, 2007, On Committees of Experts, Quarterly Journal of Economics, 122, 337-372.

Westra, W.H., J.D. Kronz, and D.W. Eisele, 2002, The Impact of Second Opinion Surgical Pathology on the Practice of Head and Neck Surgery, Head and Neck, 24, 684-693.

Williams, R.G. et al., 2007, Surgeon Information Transfer and Communication, Annals of Surgery, 245, 159-169. 